\title{
Fortaleciendo la Motivación Mediante Estrategias de Comprensión Lectora en Estudiantes de Educación Primaria
}

\author{
Jessica R. Galindo ${ }^{1}$ e Ingrid Martínez \\ Universidad Pedagógica Nacional Francisco Morazán (UPNFM), San Pedro Sula, Honduras.
}

(Enviado: Noviembre, 2014 - Publicado: Diciembre, 2014)

\begin{abstract}
Resumen:
Uno de los mayores problemas que tienen las escuelas es encontrar métodos que ayuden a sus alumnos a mejorar su aprendizaje y rendimiento académico. El propósito de esta investigación es proponer una forma de ayudar a los estudiantes con pobre rendimiento académico a mejorar sus notas mediante su involucramiento en un programa de lectura independiente significativa. Las hipótesis del estudio son: que la falta de lectura independiente significativa en los alumnos contribuye a su pobre rendimiento académico y que la asimilación de estrategias de comprensión lectora en los alumnos genera motivación hacia la lectura. Para aumentar la comprensión lectora se creó un Programa Instructivo de Lectura (PIL) y se midió la motivación de los estudiantes hacia la lectura antes y después de usar el PIL mediante el uso del Instrumento de Motivacion Intrínseca (IMI), diseñado por Ryan y Deci (2000). Se corroboró que existe una fuerte correlacion entre el rendimiento académico y la lectura independiente significativa, así como que el uso del PIL aumenta el nivel de motivación de los alumnos hacia la lectura independiente.
\end{abstract}

Palabras Claves: Lectura, Motivación, Rendimiento académico, Estudiantes.

\begin{abstract}
:
One of the biggest problems faced by schools is to find ways to help their students improve their learning and academic performance. The purpose of this research is to propose a way to help students with poor academic performance to improve their grades by becoming involved in a program of significant independent reading. The hypothesis of the study are that the lack of significant independent reading in students contributes to their poor academic performance and that the assimilation of reading comprehension strategies in students generates motivation for reading. To increase reading comprehension an Instructional Reading Program (PIL as its Spanish acronym) was created and student motivation toward reading was measured before and after using the PIL through the Intrinsic Motivation Instrument (IMI) designed by Ryan and Deci (2000). It was confirmed that there is a strong correlation between academic performance and significant independent reading and that the use of PIL increases the level of student motivation toward reading independently.
\end{abstract}

Keywords: Reading, Motivation, Academic performance, Students.

* Las autoras agradecen a Karen Hernández y Jared R. Ocampo, quienes apoyaron con información, sugerencias y análisis estadístico sin los cuales hubiera sido muy difícil llevar a cabo este trabajo de investigación y su correspondiente artículo.

\section{Introducción}

La lectura es una vía de acceso al conocimiento y es una actividad que se encuentra implicada en la mayoría de nuestras actividades diarias. Por causa de que la lectura es uno de los pilares para la adquisición y transmisión del conocimiento, es indispensable que los estudiantes adquieran el hábito de la lectura a temprana edad y reciban el correcto entrenamiento para comprender y apreciar lo que leen.

\footnotetext{
${ }^{1}$ Autor para correspondencia. Email: jessica.ocampo@gmail.com
} 
De acuerdo a Carroll (1997), los niños a temprana edad aprenden a leer utilizando un programa balanceado de lectura que incluye comprensión de letras y sonidos (fonética), así como la unión de estos sonidos para formar palabras que lleva a la decodificación y comprensión del texto que lee. Este es un proceso guiado, ya que el sentirse cómodo con su temprana familiaridad con el texto es crítico para un aprendizaje eficaz. Durante los primeros años de educación básica (primero a tercer grado), los estudiantes refuerzan su habilidad lectora; además aprenden, experimentan y practican las estrategias de lectura para convertirse en lectores independientes, comprendiendo el texto que leen. En los siguientes años de educación básica (cuarto a sexto grado), refuerzan el uso de esas estrategias, el material que leen se convierte en significativo, les da la posibilidad de convertirse en individuos capaces de aprender de manera independiente que leen, no porque tienen la habilidad para hacerlo, sino porque lo disfrutan.

Cuando aprendemos a leer y lo disfrutamos, enriquecemos nuestro vocabulario, ampliamos conocimiento y por consiguiente mejoramos nuestra capacidad de expresarnos. Sin duda alguna todo esto debe tener su efecto positivo en el rendimiento escolar académico de los estudiantes. Sin embargo, de acuerdo al Departamento de Educación de California $(1995,1996)$ "el corazón de un programa efectivo de lectura es la relación entre la instrucción sistemática de estrategias y habilidades y literatura, lenguaje y comprensión. Aun cuando las estrategias son insuficientes por sí mismas para desarrollar un buen lector, no se puede formar un buen lector sin la enseñanza de estrategias y habilidades."

\subsection{Problema de investigación}

Cuando se observan alumnos de una escuela bilingüe se piensa inmediatamente en la gama de oportunidades que estos tienen para aprender. Sin embargo, la realidad es que a pesar de todos los recursos que tienen a su disposición, varios de ellos tienen un pobre rendimiento escolar. La institución en donde se realizó este estudio ha analizado las probables causas de este bajo rendimiento escolar y como posibles soluciones para este problema, ha usado desde acomodaciones especiales (rotación de puestos en el aula, ayuda de compañeros, etc.) hasta tutorías individuales. Los resultados han sido efectivos con algunos estudiantes, pero no funcionales en todos los casos.

\subsection{Objetivos e hipótesis de investigación}

Debido a que se ha observado que en general los alumnos que tienen un efectivo rendimiento escolar son también buenos lectores, se pretende investigar si el hábito de lectura tiene una relación directa en el desempeño escolar y luego investigar cómo fomentar este hábito entre los alumnos. Entre las preguntas que se buscan contestar están: ¿Contribuye la falta de lectura al pobre rendimiento académico en los alumnos de 10 a 12 años de la escuela en estudio? Al fortalecer el uso de estrategias de lectura ¿aumenta la motivación de los alumnos hacia la lectura independiente?

\section{Objetivos generales}

- Analizar cómo la falta de hábito de lectura en los estudiantes de 10 a 12 años puede afectar su rendimiento académico.

- Evaluar cómo la asimilación de estrategias de comprensión lectora puede mejorar la motivación hacia la lectura de los estudiantes de 10 a 12 años. 


\section{Objetivos específicos}

- Identificar los estudiantes de 10 a 12 años que tienen un pobre rendimiento académico y pobre hábito lector.

- Encontrar la correlación entre falta de hábito de lectura y rendimiento académico.

- Diseñar un programa instructivo de estrategias de comprensión lectora que ayude a los alumnos de bajo rendimiento académico a aumentar su comprensión lectora.

- Evaluar la asimilación de estrategias de comprensión lectora.

- Medir la motivación alcanzada por los estudiantes hacia la lectura.

\section{Hipótesis de investigación}

1. Existe una correlación positiva alta entre el hábito de lectura independiente y el rendimiento académico.

2. La motivación de los estudiantes aumenta en la medida que aumenta su comprensión lectora.

\section{Marco Teórico}

\subsection{Rendimiento académico}

Se entiende por rendimiento académico a la evaluación del conocimiento adquirido en el ámbito escolar en un determinado espacio de tiempo. Por lo tanto un estudiante con buen rendimiento académico es aquel que obtiene calificaciones satisfactorias en las evaluaciones que debe presentar a lo largo de un año escolar. Dicho rendimiento es una medida de las capacidades y competencias del alumno, que expresa lo que éste ha aprendido a lo largo del proceso formativo, incluyendo la capacidad que tiene para responder a los estímulos educativos.

Durante mucho tiempo los educadores se han preocupado por el aprovechamiento o rendimiento escolar de sus alumnos, entendiendo que este se halla estrechamente relacionado con el proceso enseñanza - aprendizaje. Sin embargo, la forma única mediante la cual históricamente se ha medido (y en ciertos lugares todavía se mide) el rendimiento escolar, ha sido la suma de calificaciones producto del "examen" de conocimientos al que es sometido el alumno, como se nota por la definición que da Solís (2009) de rendimiento académico: "calificaciones aprobatorias o desaprobatorias que se presentan en un estudiante ante un determinado curso". Esta forma de medir que se sesgaba hacia el intelecto se convirtió en norma, principio y fin, de manera que al alumno se le solicitaba que repitiese letra por letra lo aprendido, formando de esta manera buenos entes repetidores, pero no pensadores ni hacedores.

Sin embargo, los nuevos conceptos de rendimiento académico se han inclinado por la idea de que la educación debe ir centrada en el alumno y no en el maestro, en el aprendizaje y no en la enseñanza. El resultado de esta inclinación ha sido la gran gama de diferentes tipos de evaluaciones que ahora se pueden aplicar a los alumnos para medir su verdadero aprovechamiento. Esto implica que ya no se tiene una única manera de evaluar el rendimiento mediante pruebas escritas que evalúan solo conocimiento adquirido, sino también se usan otro tipo de evaluaciones que miden aptitudes y competencias desarrolladas (Carrasco, 1985). 
Los especialistas opinan que son varios los factores que afectan el satisfactorio aprovechamiento educativo de los alumnos. Entre ellos se encuentran la metodología de enseñanza utilizada por el docente, el apoyo que el alumno recibe en la casa, las habilidades del estudiante como individuo único, el grado de dificultad en algunas materias, etc. Otras cuestiones están directamente relacionadas al factor psicológico, tales como la ansiedad, la poca motivación, el desinterés o las distracciones en clase, las cuales dificultan la comprensión de los conocimientos impartidos por el docente y termina afectando al rendimiento académico a la hora de las evaluaciones. Por otra parte, el rendimiento académico también puede estar condicionado a los hábitos de estudio que tiene el alumno, a la subjetividad del docente cuando corrige exámenes, o al sesgo hacia él que puede producirse por situaciones actitudinales del alumno.

\subsection{La lectura y sus beneficios}

Una de las herramientas que puede ser usada para potenciar el rendimiento académico es la lectura. En este mundo tan acelerado en el cual vivimos es indispensable mantenerse informado de tantos nuevos descubrimientos y aportaciones hechas por un sin número de científicos y personas dedicadas a la investigación. De acuerdo a Molina (2006) ninguna institución educativa puede conformarse con trasmitir los conocimientos actuales, sino que debe preparar a los alumnos para que continúen aprendiendo una vez que han dejado la escuela. Esto exige formar lectores y escritores autónomos. Para los alumnos ese hábito representará uno de los aprendizajes de mayor trascendencia y proyección futura, ya que este ejercicio lector modelará el talante cívico y cultural de su persona.

Cuando un alumno adquiere el hábito de la lectura incrementa su capacidad analítica, ya no es solamente la unión de palabras y comprensión de oraciones simples, sino que aprende a discernir la otra información que el escritor espera que el lector infiera. Cuando un alumno no es capaz de entender o analizar lo que el texto dice, es decir analizar su contenido esto le conduce a frustración, aburrimiento, desinterés y por ende al fracaso escolar. Los estudiantes que se convierten en lectores son personas inquisitivas, que estimulan sus propios intereses, satisfacen sus propias ansias y se despierta en ellos el deseo de conocer otros mundos ajenos al suyo.

La labor de un maestro a fin de fomentar este hábito no se limita a asignar libros para lectura independiente, asignando un puntaje, sino llevarle de la mano y despertar su interés a través de la enseñanza de estrategias que permitan al alumno mismo desmenuzar la información y digerirla placenteramente. Si el alumno logra este cometido irá adquiriendo su propio gusto literario, definiendo su marco literario de referencia, así como sus propios clásicos.

Además de los beneficios antes mencionados, se pueden agregar otros:

- Expansión de vocabulario - El estudiante adquiere un vocabulario nutrido que ejercita al momento de hablar o escribir.

- Fortalecimiento de la memoria - El lector aprende datos o hechos importantes nuevos forma nuevas conexiones en el cerebro y fortalece las ya existentes.

- Incrementa la capacidad de concentración - Al leer un libro el lector se mantiene enfocado en la historia que lee, el resto de su entorno toma menos. El lector se centra en los detalles que trata de absorber. 
De acuerdo a Richard Allington (2012), antiguo presidente de la Asociación Internacional de Lectura, existe una relación directa entre la cantidad de material leído y resultados finales favorables de exámenes de lectura. De acuerdo a su investigación, un niño de quinto grado debe leer un promedio de 40-45 minutos al día para obtener puntajes en el percentil 90 en exámenes estandarizados de lectura. Estos niños leen un promedio de 2 millones 357 mil palabras al año. Un alumno de quinto grado que lee sólo un promedio de 13 minutos al día se podría encontrar en el percentil 10 en el mismo examen estandarizado, ya que sólo lee 51 mil palabras al año. El tiempo que un niño dedica a la lectura le trae beneficios duraderos.

\subsection{Estrategias de comprensión lectora}

Se podría definir que una estrategia es la capacidad de pensar de tal forma que un individuo ordene su curso de acción para que le permita avanzar con eficacia. Según Nisbet y Shucksmick (1987) las estrategias son procesos ejecutivos, además de capacidades cognitivas de orden más elevado, estrechamente relacionadas con la metacognición, es decir la capacidad de conocer el propio conocimiento, de pensar sobre nuestra actuación, de planificarla y que permiten controlar y regular la actuación inteligente. Es definitivamente necesario que el estudiante conozca estrategias que le permitan digerir información, separarla, procesarla para luego relacionarla con datos que ya conoce.

Las estrategias de lectura le permiten al alumno planificar la tarea total de la lectura, su propia ubicación, es decir su motivación y disponibilidad ante ella, además de facilitar su comprensión, revisión y control de lo que se lee, toma de decisiones adecuada en función de los objetivos que se persiguen (Solé, 1992). Solé (1992) incluye las siguientes estrategias de lectura como las más importantes:

- Activar y aportar a la lectura conocimientos previos. El estudiante se hace las siguientes interrogantes: ¿Qué sé yo acerca del contenido del texto? ¿Qué sé acerca de contenidos afines que me puedan ser útiles? ¿Qué otras cosas sé que puedan ayudarme: acerca del autor, del género, del tipo de texto?

- Hacer preguntas a lo largo del proceso de lectura. El alumno se cuestiona a sí mismo, monitoreando de manera individual el contenido de lo que lee, formulándose preguntas como: ¿Qué tengo que leer? ¿Por qué/para qué tengo que leerlo? ¿Qué pasa después? ¿Cómo llegue a este punto? ¿Quién? ¿Cómo? ¿Por qué? ¿Cuándo? ¿Qué pasaría si?

- Evaluar la consistencia interna del contenido. Expresa el texto y su compatibilidad con el conocimiento previo, y con lo que dicta el «sentido común». ¿Tiene sentido este texto? ¿Presentan coherencia las ideas que en él se expresan? ¿Discrepa abiertamente de lo que yo pienso, aunque sigue una estructura argumental lógica? ¿Se entiende lo que quiere expresar? ¿Qué dificultades plantea?

- Sintetizar/ Resumir. Comprobar continuamente si la comprensión tiene lugar mediante la revisión y recapitulación periódica y la autointerrogación ¿Qué se pretendía explicar en este párrafo -apartado, capítulo-? ¿Cuál es la idea fundamental que extraigo de aquí? ¿Puedo reconstruir el hilo de los argumentos expuestos? ¿Puedo reconstruir las ideas contenidas en los principales apartados? ¿Tengo una comprensión adecuada de los mismos? 
- Elaborar y probar inferencias. Es decir de diversos tipos como interpretaciones, hipótesis y predicciones y conclusiones, haciéndose el estudiante las siguientes preguntas: ¿Cuál podrá ser el final de esta novela? ¿Qué sugeriría yo para solucionar el problema que aquí se plantea? ¿Cuál podría ser -tentativamente- el significado de esta palabra que me resulta desconocida? ¿Qué le puede ocurrir a este personaje?

\subsection{El docente y las enseñanzas de estrategias de comprensión lectora}

La comprensión y las estrategias de lectura van de la mano. Estas son las herramientas necesarias para hacer funcionar ese proceso vital en la mente del estudiante. Según la perspectiva constructivista de varios autores (Edwards y Mercer, 1988; Coll, 1990; Rogoff, 1984), el llevar al estudiante a comprender la lectura es un proceso de construcción conjunto en el cual el maestro sirve de guía, es decir, debería asegurarse que el alumno comprenda las estrategias y sepa aplicarlas correctamente. Se habla de participación guiada (Rogoff, 1984) en la cual el maestro le permite al estudiante mediante un proceso dirigido: primero, relacionar su conocimiento previo y necesario para abordar dicha situación; segundo, brindarle una visión o estructura general para llevar a cabo su tarea, y finalmente permite al estudiante asumir la responsabilidad de su desarrollo de manera progresiva, hasta que este se muestre competente al aplicar de manera autónoma las estrategias aprendidas.

Para ampliar un poco la importancia del docente y su participación en este proceso de enseñanza y aprendizaje de estrategias vitales para la comprensión, Bruner y sus colaboradores (Wood, Bruner y Ross, 1976) hablan de un proceso de andamiaje, que describe metafóricamente como debería ser el proceso de enseñanza-aprendizaje: los andamios son imprescindibles en el proceso de construcción; estos se sitúan un poco más elevados del edificio que se construirá. Los retos que constituyen la enseñanza deben ir un poco más allá de lo que los estudiantes sean capaces de resolver. El docente dirige la presentación de estos retos y monitorea al alumno que trata de resolverlos. De hecho, una vez construido el edificio, el andamio se retira y no queda rastro de él, ni el edificio se derrumba sin él. Del mismo modo, una vez que el alumno ha desarrollado la competencia de resolver retos y controlar su propio aprendizaje, las ayudas que caracterizan la enseñanza se retiran progresivamente. La buena enseñanza por tanto, no es sólo la que se sitúa un poco más allá del nivel actual del estudiante, sino la que asegura la interiorización de lo que se enseñó y su uso autónomo por parte de aquel.

\subsection{Relación entre motivación y comprensión lectora}

Se puede definir la motivación como una fuerza que energiza, marca el curso y guía las prácticas de los alumnos. A menudo vemos la motivación de los estudiantes reflejada en una inversión personal y un compromiso cognitivo, emocional y conductual en sus actividades escolares (Fredericks, Blumenfeld, y Paris, 2004). De acuerdo a Maehr y Meyer (1997), la motivación marca la pauta en el comportamiento de las personas hacia metas particulares, por lo tanto dicha motivación afecta las elecciones que los estudiantes hacen. Un estudiante motivado incrementa la cantidad de esfuerzo y energía que dedica a actividades directamente relacionadas con sus necesidades y metas (Pintrich et al., 1993). Por lo tanto la motivación determina si los alumnos cumplen con una tarea con entusiasmo y sin reservas o con indiferencia y desinterés.

Según Larson (2000) y Wigfield (1994) la motivación también incrementa la persistencia que los estudiantes exhiben para completar las actividades que inician, a pesar de verse 
frustrados en el proceso por problemas o por la dificultad de la tarea. Esta motivación es definitivamente necesaria en el proceso que lleva a un alumno desde aprender nombres y sonidos de letras, decodificación, hasta la comprensión autónoma y efectiva de lo que lee. La enseñanza de estrategias de comprensión lectora hace más fácil este proceso, pues el alumno toma estas herramientas y logra entender lo que se le presenta en forma de texto. Un alumno que sabe lo que hace, comprende lo que lee y es un alumno motivado; como ha dominado este proceso llega a disfrutarlo y a necesitarlo. Así se forma el hábito de lectura, cuando un estudiante entiende y disfruta de lo que lee, por tanto quiere leer más para sentirse satisfecho.

De acuerdo a algunos investigadores (Aunola, Leskinen, Onatsu-Arvilomni y Nurmi, 2002) los alumnos se desmotivan cuando no aprenden correctamente las estrategias de comprensión lectora. Esta situación empeora porque se dan otras acciones derivadas de esta falta de adquisición de herramientas para la comprensión lectora. Estas acciones llevan al alumno a la desmotivación, problemas cognitivos y conductuales (Stanovich, 1986), este no se interesa por leer y llega a una situación donde no se obtiene el aprendizaje esperado. Por tanto una pobre motivación es el posible resultado de no conocer las estrategias elementales para la comprensión lectora y de no poseer la costumbre de leer prolongadamente.

Una de las formas más utilizadas para medir la motivación es el uso de la teoría de autodeterminación de Edward Decy y Richard Ryan (2000). Dicha teoría propone medir el nivel de motivación de los estudiantes mediante un instrumento que tiene siete subescalas. La subescala de interés/disfrute del instrumento es considerada la medición de la motivación intrínseca. Sin embargo, las subescalas de elección percibida (el estudiante está haciendo la actividad por su propia elección) y competencia percibida (el estudiante siente que es competente en la actividad) son predictores positivos de la motivación, así como la subescala de presión/tensión experimentada es un predictor negativo de la motivación (Hassandra, M., Goudas, M. y Chroni, S., 2003).

\section{Metodología}

El proyecto de investigación se llevó a cabo en una escuela bilingüe, enfocándose en los alumnos de 10 a 12 años (5 y 6 grado). Se utilizó la herramienta metodológica de investigación-acción. Dicho instrumento conecta el enfoque experimental con programas de acción que responden a problemas sociales. Este proceso es reflexivo, permite al investigador observar cambios, documentarlos y trabajar en base a esas observaciones.

\subsection{Operacionalización de las variables}

Para poder responder las preguntas de investigación establecidas, probar las hipótesis planteadas y lograr los objetivos propuestos, se operativizaron las variables de estudio como se muestra en la Tabla 1.

\section{Lectura}

Recolección de datos sobre los hábitos de lectura independiente de los estudiantes. Se usó el "Reading Log", que es un documento en donde se registra cuánto tiempo lee el estudiante diariamente. Se espera que el estudiante lea por lo menos 45 minutos al día. Al final de 8 semanas se sumaron los días que cumplieron con su asignación de lectura, contabilizándolos como días para efecto de uso práctico de variables. 
Tabla 1. Operativización de las variables

\begin{tabular}{|c|c|c|c|c|}
\hline Variable & Tipo de Variable & Operacionalización & Indicador & Valor \\
\hline Lectura & $\begin{array}{l}\text { Cuantitativa } \\
\text { discreta } \\
\text { independiente }\end{array}$ & $\begin{array}{c}\text { Valorización cuantitativa de la } \\
\text { dedicación a la lectura } \\
\text { independiente a través de } \\
\text { Reading Log. }\end{array}$ & Número de días & \begin{tabular}{|l} 
La cantidad total de \\
días cumplidos de \\
lectura independiente \\
(> 45 minutos) en el \\
transcurso de 8 \\
semanas. \\
\end{tabular} \\
\hline $\begin{array}{l}\text { Rendimiento } \\
\text { académico }\end{array}$ & $\begin{array}{l}\text { Cuantitativa } \\
\text { continua } \\
\text { dependiente }\end{array}$ & $\begin{array}{l}\text { Combinación de examen de } \\
\text { vocabulario y la nota de } 1 \mathrm{er} \\
\text { parcial de la clase de } \\
\text { Reading. Esto con una } \\
\text { ponderación de } 70 \% \text { (nota } \\
\text { Reading) y } 30 \% \text { (nota de } \\
\text { examen de vocabulario) }\end{array}$ & Porcentaje & $\begin{array}{l}\text { Nota ponderada entre } \\
0-100 \text { en donde } 100 \\
\text { es la nota máxima. }\end{array}$ \\
\hline Estrategias & $\begin{array}{c}\text { Cuantitativa } \\
\text { independiente }\end{array}$ & $\begin{array}{c}\text { Asimilación de estrategias de } \\
\text { comprensión lectora que será } \\
\text { medible a través de } \\
\text { exámenes de diagnóstico } \\
\text { aplicados al principio del PIL, } \\
\text { después de } 4 \text { semanas y al } \\
\text { final del PIL (8 semanas). }\end{array}$ & Porcentaje & $\begin{array}{c}\text { Notas de exámenes } \\
\text { diagnósticos que se } \\
\text { realicen a través del } \\
\text { PIL (nota entre } 0 \text { y } \\
100 \text { pts, en donde } \\
100 \text { es comprensión } \\
\text { total de todos los } \\
\text { temas evaluados). }\end{array}$ \\
\hline Motivación & $\begin{array}{c}\text { Cuantitativa } \\
\text { dependiente ordinal }\end{array}$ & $\begin{array}{l}\text { Se hará por medio de } \\
\text { Instrumento de Motivación } \\
\text { Intrínseca (IMI) }\end{array}$ & Valor promedio & $\begin{array}{c}\text { Valor entre } 1 \text { y } 5 \text { de } \\
\text { una escala Likert }\end{array}$ \\
\hline
\end{tabular}

\section{$\underline{\text { Rendimiento académico }}$}

Aplicación de un Examen diagnóstico inicial (70\%) de estrategias de comprensión lectora que incluyen las mencionadas en el Marco Teórico. Además se aplicó también un Examen estandarizado de vocabulario (30\%) de ESP Publishers (2001) para conocer el nivel de vocabulario que los alumnos tenían. La combinación de las notas de ambos exámenes (Diagnóstico inicial y Vocabulario) da el rendimiento académico. La ponderación entre ambos exámenes y su valor se llevó a través de un consenso de las maestras que forman parte del equipo de investigación. Una calificación menor al $70 \%$ se consideró reprobado.

\section{Selección}

Los estudiantes que formarán parte del Programa Instructivo de Lectura (PIL) fueron elegidos a través de:

- Pobre rendimiento académico. Su calificación en la ponderación final del Examen diagnóstico inicial y Examen estandarizado de vocabulario fue menor al $70 \%$.

- Entrevistas a maestras anteriores y orientadora de la institución para conocer cuáles de los alumnos con nota inferior a $70 \%$ tienen pobre rendimiento académico debido a problemas de motivación. Se registraron estos comentarios en la hoja Perfil del Alumno.

- Se invitó a los alumnos participantes del PIL a un grupo focal para indagar sobre su motivación hacia la lectura además de aplicarles el IMI (Instrumento de Motivación Intrínseca). Estas observaciones se documentaron en el Diario del docente. Los alumnos se reunieron nuevamente en un grupo focal al finalizar el PIL para hacer las comparaciones (motivación) correspondientes. El IMI se volvió a aplicar. 
- Previo al PIL, se realizó en las instalaciones una reunión informativa con los padres de los alumnos participantes. Se les explicó que el objetivo de dicho programa seria reforzar el uso de estrategias de comprensión lectora en sus hijos, a fin de motivarles a adquirir el hábito de la lectura además de mejorar su rendimiento académico. Los padres se comprometieron en apoyar a sus hijos, permitiéndoles quedarse 45 minutos después del horario normal escolar mientras dure el programa.

\section{Motivación}

Se mide a través del Instrumento de Motivación Intrínseca (IMI) propuesto por Edward Decy y Richard Ryan para determinar el nivel de motivación de los estudiantes a través las experiencias subjetivas de un participante en una actividad meta. El instrumento tiene siete subescalas, pero solo cuatro de ellas son usadas para medir la motivación intrínseca. En este experimento solo se emplearán dos de ellas: interés/disfrute y presión/tensión experimentada. El instrumento fue adecuado a la actividad de lectura independiente de los alumnos (ver Figura 2) y está basado en una escala Likert de 7 puntos ( 1 = not true at all, $4=$ somewhat true, $7=$ very true)

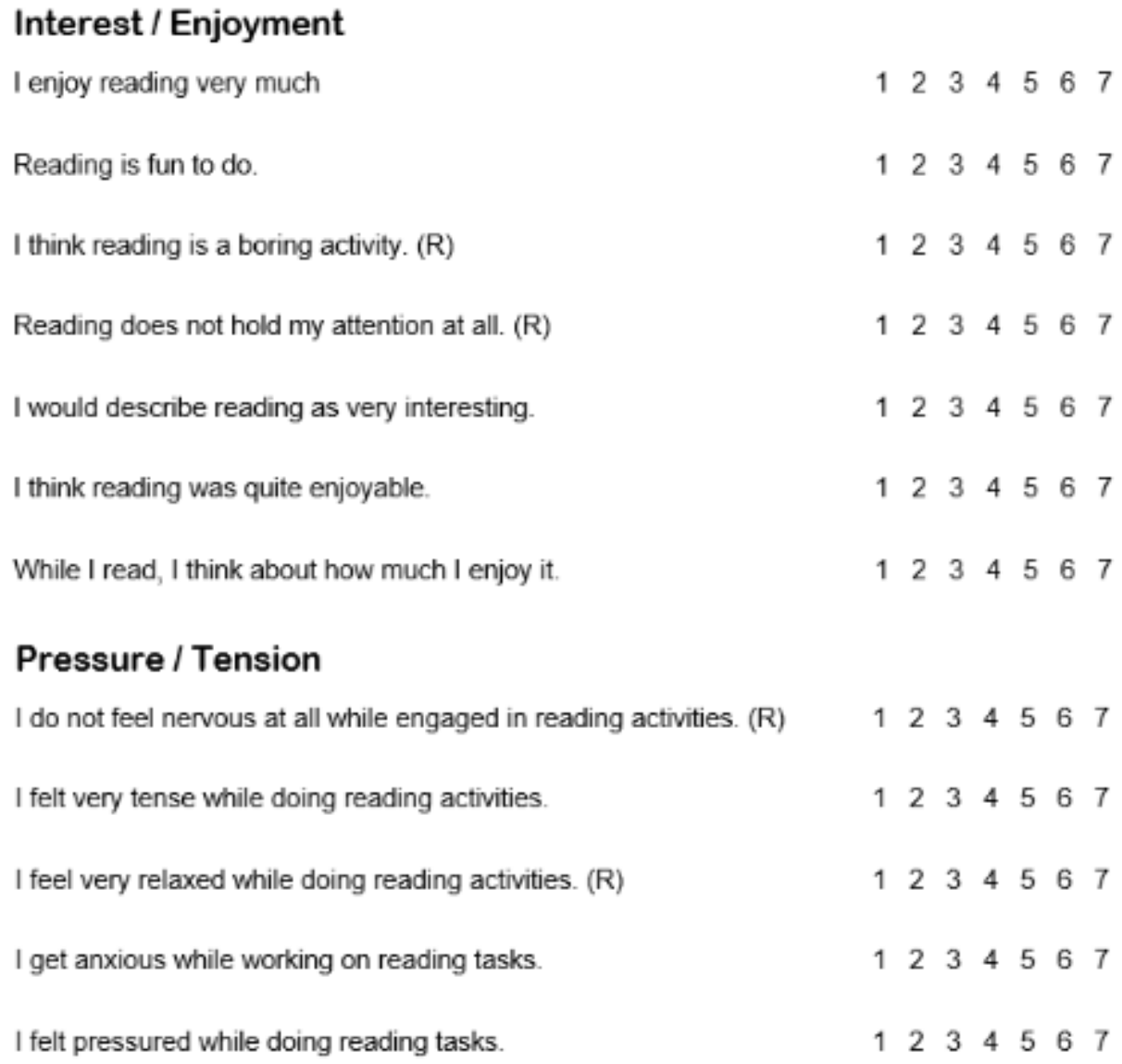

Figura 1. Instrumento de motivación intrínseca a la lectura independiente

\section{Estrategias de comprensión lectora}

Para aumentar la motivación se dió reforzamiento de estrategias de comprensión lectora utilizando el Programa Instructivo de Lectura (PIL), con una duración de 8 semanas. Para dicho programa se escogieron 5 de las estrategias más importantes y se elaboró un 
programa de historias y actividades. Las clases se impartieron 3 veces a la semana con una duración de 45 minutos cada una. Se llevó el Diario del docente para el tipo de metodología que se estaba utilizando. Este fue llenado por las personas encargadas de dar las clases del PIL. Solamente participaron en esta parte del proyecto las integrantes del grupo de investigación que laboraban para la institución, en seguimiento a las políticas internas del centro educativo; sin embargo, semanalmente el grupo completo de investigación evaluó avances o comentarios. Cada alumno, además de su hoja de perfil, tenía su propio registro con resumen de sus avances, observaciones y cambios individualizados que se hicieron en la enseñanza del PIL a fin de lograr el objetivo propuesto. Cada estudiante tuvo un cuaderno interactivo que dejaba constancia de actividades realizadas en clases.

Se indagó sobre la mejoría del nivel de comprensión lectora a través de la aplicación de exámenes diagnósticos estandarizados, el primero después de 4 semanas (Mid-examen diagnóstico) y al final del PIL (Post-examen diagnóstico).

\subsection{Comprobación de las hipótesis}

Para comprobar la primera hipótesis se hizo un análisis de correlación entre los días de asignaciones cumplidas de lectura independiente y el desempeño de cada estudiante. Si existe una correlación positiva alta se sabe que a mayor cantidad de horas de lectura, mayor el rendimiento académico (y por ende a menor lectura independiente menor rendimiento académico).

Para efectos de comprobar la segunda hipótesis, a los alumnos de pobre rendimiento académico se les aplicó el IMI al comienzo del PIL y se obtuvo un promedio de su motivación a través de las dimensiones de interés/disfrute y presión/tensión. Se repitió este mismo proceso una última vez al grupo bajo estudio. En este caso la hipótesis se comprobará si se observa un aumento en los promedios de la segunda medición en contraste con los de la primera medición antes de que el PIL se llevará a cabo en el caso de la dimensión interés/disfrute y una disminución en la dimensión presión/tensión.

\section{Análisis y Resultados}

La información durante todo el proceso fue registrada, tabulada y finalmente graficada. El análisis de correlación permite revisar la fuerza de la relación lineal entre el número de días en que los alumnos cumplieron su asignación de lectura y su rendimiento académico. Por lo tanto la hipótesis que la falta de lectura contribuye al pobre rendimiento académico en los alumnos, podría ser pensada de la siguiente forma:

$$
\begin{aligned}
& H_{0}: r_{\text {lectura y rend. acad. }} \leq 0.70 \\
& H_{1}: r_{\text {lectura y rend. acad. }}>0.70
\end{aligned}
$$

Como se observa en la Figura 2, el análisis de correlación arroja un valor de 0.78 , lo que implica que la relación es positiva, o sea directamente proporcional (a mayor número de días de lectura mayor rendimiento). A pesar de que no es una correlación perfectamente positiva, sí se encuentra dentro del rango que puede ser considerado muy alto, especialmente si se considera que son datos relacionados con seres humanos que normalmente presentan gran variabilidad.

Para terminar de validar estos resultados se calculó el coeficiente de determinación (también conocido como r cuadrado). El valor obtenido fue de 0.62 (ver Figura 2) lo que puede interpretarse como que el número de días de lectura significativa puede explicar en 
un $62 \%$ el rendimiento académico obtenido por los estudiantes. Esto tiene sentido, ya que leer por lo menos 45 minutos cada día, además de mejorar el vocabulario y expandir la mente; también demuestra disciplina y perseverancia, que son características necesarias para tener éxito académico.

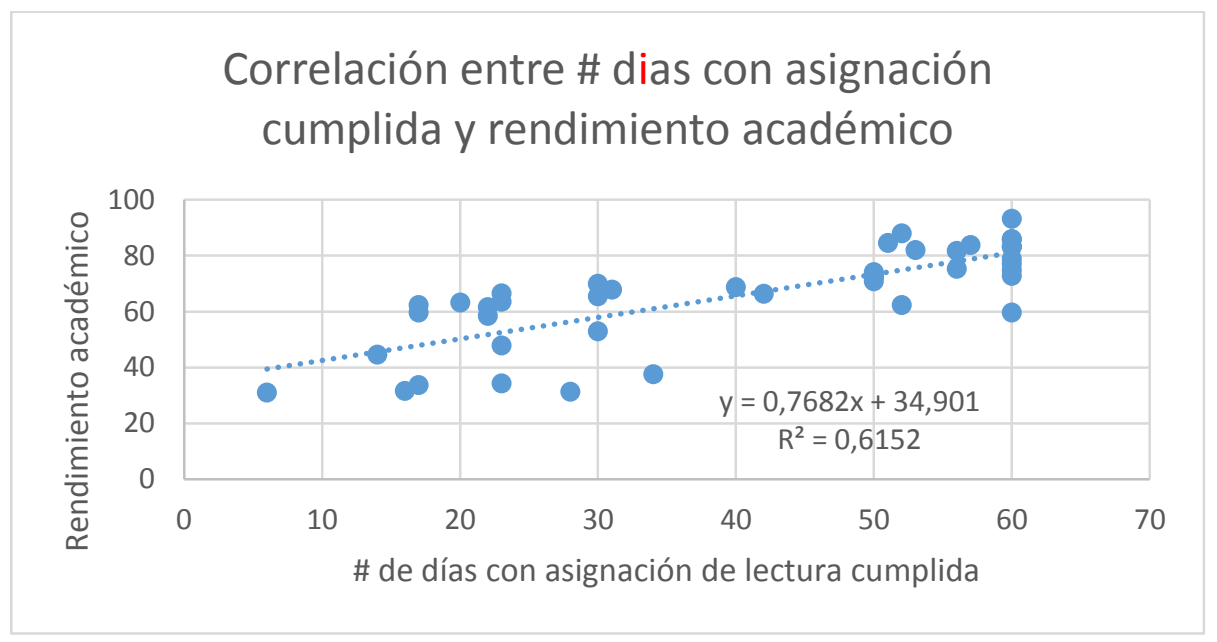

Figura 2. Correlación entre días con lectura cumplida y rendimiento académico

Por lo tanto, basados en los resultados del análisis de correlación y su correspondiente coeficiente de determinación, nuestra hipótesis que la falta de lectura contribuye al pobre rendimiento académico en los alumnos de 10 a 12 años de la escuela bilingüe en estudio, es verdadera, ya que existe una correlación positiva entre el rendimiento y la lectura.

Considerando que el instrumento propuesto por Ryan y Deci para medir la motivación de un grupo de personas (Intrinsic Motivation Inventory), le permite medir 7 dimensiones, se decidió usar las dos que mejor describen la motivación en estudiantes de educación primaria: el interés o disfrute al realizar una actividad (en este caso la lectura) y la presión o tensión experimentada por causa de la misma actividad. Esto implica que para corroborar la hipótesis que la asimilación de estrategias de comprensión lectora de los alumnos de pobre rendimiento académico genera motivación en la lectura, es necesario hacer dos pruebas de hipótesis simultáneas:

1. El interés o disfrute (ID) de los estudiantes aumentó luego de que los estudiantes participaron en el Programa Instructivo de Lectura (PIL), es decir

$$
\begin{aligned}
& H_{0}: \mu_{I D \text { antes }}-\mu_{I D \text { después }} \geq 0 \\
& H_{1}: \mu_{I D \text { antes }}-\mu_{I D \text { después }}<0
\end{aligned}
$$

2. La presión/tensión (PT) de los estudiantes disminuyó luego de que los estudiantes participaron en el Programa Instructivo de Lectura (PIL), es decir

$$
\begin{aligned}
& H_{0}: \mu_{P T \text { antes }}-\mu_{P T \text { después }} \leq 0 \\
& H_{1}: \mu_{P T \text { antes }}-\mu_{P T \text { después }}>0
\end{aligned}
$$

Para hacer esta prueba de hipótesis se les colocó a los alumnos en estudio (pobre rendimiento académico) el instrumento que puede verse en la Figura 1, el cual fue diseñado de acuerdo al propuesto por Ryan y Deci. Esto se hizo antes que ellos 
participaran en el PIL y después de haberlo concluido. Los resultados de dichas encuestas pueden verse en las tablas 2 (antes) y 3 (después).

Tabla 2. Resultado de la encuesta a alumnos antes de aplicar el PIL

Encuesta antes de aplicar el PIL

\begin{tabular}{|c|c|c|c|c|c|c|c|c|c|c|}
\hline & \multicolumn{10}{|c|}{ INTEREST/ENJOYMENT } \\
\hline Alumno & P1 & P2 & P3 & P3R & P4 & P4R & P5 & P6 & P7 & TOTAL \\
\hline Dora & 4 & 5 & 1 & 7 & 3 & 5 & 5 & 6 & 3 & 35 \\
\hline Pablo & 4 & 3 & 3 & 5 & 4 & 4 & 2 & 3 & 2 & 23 \\
\hline Eddie & 4 & 5 & 1 & 7 & 1 & 7 & 5 & 4 & 3 & 35 \\
\hline Marianne & 4 & 3 & 1 & 7 & 7 & 1 & 5 & 4 & 2 & 26 \\
\hline Pablo M. & 2 & 1 & 2 & 6 & 3 & 5 & 4 & 2 & 1 & 21 \\
\hline Marco & 2 & 2 & 6 & 2 & 3 & 5 & 4 & 3 & 2 & 20 \\
\hline Abner & 2 & 4 & 3 & 5 & 2 & 6 & 1 & 3 & 2 & 23 \\
\hline Margarita & 3 & 3 & 5 & 3 & 5 & 3 & 1 & 4 & 3 & 20 \\
\hline Natalia & 4 & 3 & 5 & 3 & 4 & 4 & 4 & 3 & 3 & 24 \\
\hline & & & & & & & & & & 25,22 \\
\hline & & & & & & ai & in Es & & dar & 5,87 \\
\hline
\end{tabular}

\begin{tabular}{|c|c|c|c|c|c|c|c|}
\hline \multicolumn{8}{|c|}{ PRESSURE/TENSION } \\
\hline P1 & P1R & P2 & P3 & P3R & P4 & P5 & TOTAL \\
\hline 7 & 1 & 6 & 7 & 1 & 1 & 1 & 10 \\
\hline 3 & 5 & 5 & 2 & 6 & 3 & 2 & 21 \\
\hline 2 & 6 & 4 & 3 & 5 & 5 & 6 & 26 \\
\hline 4 & 4 & 5 & 2 & 6 & 6 & 6 & 27 \\
\hline 2 & 6 & 4 & 2 & 6 & 5 & 4 & 25 \\
\hline 3 & 5 & 4 & 3 & 5 & 6 & 2 & 22 \\
\hline 3 & 5 & 2 & 1 & 7 & 3 & 7 & 24 \\
\hline 3 & 5 & 5 & 4 & 4 & 3 & 4 & 21 \\
\hline 4 & 4 & 6 & 3 & 5 & 3 & 5 & 23 \\
\hline \multicolumn{7}{|c|}{ Promedio } & 22,11 \\
\hline \multicolumn{7}{|c|}{ Desviación Estándar } & 5,01 \\
\hline
\end{tabular}

Tabla 3. Resultado de la encuesta a alumnos después de aplicar el PIL

Encuesta después de aplicar el PIL

\begin{tabular}{|c|c|c|c|c|c|c|c|c|c|c|}
\hline & \multicolumn{10}{|c|}{ INTEREST/ENJOYMENT } \\
\hline Alumno & P1 & P2 & P3 & P3R & P4 & P4R & P5 & P6 & P7 & TOTAL \\
\hline Dora & 6 & 5 & 2 & 6 & 1 & 7 & 5 & 4 & 4 & 37 \\
\hline Pablo & 6 & 5 & 2 & 6 & 1 & 7 & 5 & 4 & 4 & 37 \\
\hline Eddie & 4 & 5 & 2 & 6 & 1 & 7 & 6 & 5 & 4 & 37 \\
\hline Marianne & 4 & 3 & 2 & 6 & 5 & 3 & 6 & 5 & 5 & 32 \\
\hline Pablo M. & 3 & 3 & 4 & 4 & 3 & 5 & 4 & 4 & 2 & 25 \\
\hline Marco & 2 & 6 & 4 & 4 & 3 & 5 & 3 & 4 & 5 & 29 \\
\hline Abner & 3 & 4 & 4 & 4 & 3 & 5 & 2 & 3 & 3 & 24 \\
\hline Margarita & 4 & 4 & 5 & 3 & 3 & 5 & 4 & 4 & 4 & 28 \\
\hline Natalia & 6 & 5 & 5 & 3 & 5 & 3 & 6 & 6 & 7 & 36 \\
\hline & & & & & & & & & & 31,67 \\
\hline & & & & & & & n Es & stán & & 5,34 \\
\hline
\end{tabular}

\begin{tabular}{|c|c|c|c|c|c|c|c|}
\hline \multicolumn{8}{|c|}{ PRESSURE/TENSION } \\
\hline P1 & P1R & P2 & P3 & P3R & P4 & P5 & TOTAL \\
\hline 5 & 3 & 2 & 5 & 3 & 4 & 2 & 14 \\
\hline 5 & 3 & 2 & 5 & 3 & 4 & 2 & 14 \\
\hline 4 & 4 & 6 & 3 & 5 & 4 & 5 & 24 \\
\hline 6 & 2 & 2 & 7 & 1 & 5 & 5 & 15 \\
\hline 4 & 4 & 4 & 3 & 5 & 3 & 4 & 20 \\
\hline 4 & 4 & 3 & 3 & 5 & 4 & 3 & 19 \\
\hline 6 & 2 & 4 & 6 & 2 & 3 & 5 & 16 \\
\hline 4 & 4 & 3 & 4 & 4 & 4 & 5 & 20 \\
\hline 3 & 5 & 4 & 5 & 3 & 3 & 3 & 18 \\
\hline \multicolumn{7}{|c|}{ Promedio } & 17,78 \\
\hline \multicolumn{7}{|c|}{ Desviación Estándar } & 3,35 \\
\hline
\end{tabular}

Considerando que se contaba con una muestra de 9 alumnos (muestra pequeña), se usó una prueba t para dos muestras de varianzas separadas para comparar estadísticamente el antes y el después de los resultados obtenidos en el interés o disfrute (ID) y en la presión tensión (PT).

Usando un $95 \%$ de confianza, en el caso del interés o disfrute, el valor de p obtenido fue de 0,004 que claramente está en el área de rechazo (0.05), por lo que se rechaza la hipótesis nula y se acepta la hipótesis sugerida, que el interés o disfrute después del PIL es mayor que antes del mismo. En el caso de la presión o tensión, siempre usando un 95\% de confianza, se obtuvo un valor p de 0,0004 que igualmente cae en el área de rechazo. Por lo tanto también se puede aceptar la hipótesis que la presión o tensión disminuye en los alumnos una vez que han participado del PIL. 
Por lo tanto la hipótesis original planteada que la asimilación de estrategias de comprensión lectora de los alumnos de pobre rendimiento académico genera motivación en la lectura, ha sido comprobada como correcta.

\section{Conclusiones}

En este estudio se corroboró que existe una relación entre el rendimiento académico y la lectura independiente significativa. Además se confirmó que el uso de PIL (Programa Interactivo de Lectura) diseñado por las investigadoras aumenta el nivel de motivación de los alumnos hacia la lectura independiente.

Se sugiere hacer trabajos futuros para encontrar si existe una correlación entre los resultados del PIL (asimilación de competencias de comprensión lectora) y el aumento de la lectura independiente significativa ( $40-45$ minutos). Tambien se sugiere que este estudio se haga, considerando aplicar el PIL durante un periodo más prolongado con el fin de que los alumnos desarrollen las competencias de comprensión lectora de manera eficaz y completa.

\section{Bibliografía}

Allington, R. L., 2012. "What really matters for struggling readers: Designing researchbased programs" (3rd ed.). Boston: Allyn and Bacon

Aunola, K., Leskinen, E., Onantsu-Arvilommi, T., y Nurmi, J., 2002. "Three methods for studying developmental change: A case of reading skills and self-concept”. Brithish Journal of Educational Psychology, Vol. 72, pp. 343-364.

Carroll, V., 1997. "Learning to Read, Reading to Learn". Resources in Education, recuperado de http://eric.ed.gov/ [Consulta Julio 2014]

Carrasco, J., 1985. “La Recuperación Educativa”. Madrid. Anaya.

Coll, C., 1992. "Constructivismo e intervención educativa". Aula de Innovación Educativa. Recuperado de http://www.grao.com/revistas/aula/003-didactica-delos-procedimientos--distintas-formas-de-elaboracion-y-gestion/constructivismo-eintervencion-educativa

Departamento de Educación de California (1995, 1996). Recuperado de http://www.ericdigests.org/2000- 3/reading.htm

Edwards, D. y Mercer, N., 1988. "El conocimiento compartido. El desarrollo de la comprensión en el aula". Barcelona, Paidós/MEC.

Fredericks, J.A., Blumenfeld, P.C. y Paris, A.H., 2004. "School Engagement: Potential of the Concept, State of the Evidence”. Review of Educational Research, Vol. 74, pp. 59-109. 
Hassandra, M., Goudas, M. y Chroni, S., 2002. "Examining Factors Associated with Intrinsic Motivation in Physical Education: A Qualitative Approach”. Psychology in Sports and Exercise. Vol. 4, pp. 211-223.

Larson, R.W., 2000. "Toward a psychology of positive youth development". The American Psychologist, Vol. 55, pp. 170-183.

Maehr, M.L. y Meyer, H.A., 1997. "Understanding Motivation and Schooling: Where we've been, where we are, and where we need to go". Educational Psychology Review, Vol. 9, pp.371-409.

Molina V. L., 2006. “Lectura y educación, los hábitos lectores y su repercusión académica en la ESO” Revista Ocnos, pp.103-120.

Nisbet y Shucksmick, 1987, “Estrategias de lectura”. Editorial Graó. Barcelona

Pintrich, P.R., Smith, D.A., Garcia, T. y Mckeachie, W.J., 1993. "Reliability and predictive validity of the motivated strategies for learning questionnaire (MSLQ)”. Educational and Psycological Measurement, Vol. 53, pp. 801-803.

Ryan, R. M. y Deci, E. L., 2000. "Intrinsic and Extrinsic Motivations: Classic Definitions and New Directrios”. Contemporary Educational Psychology, Vol. 25, pp. 54-67.

Solé, I., 1992. "Estrategias de lectura”. Barcelona, Editorial Grao.

Solis, J.C., 2009. Recuperado de http://psicopedagogiaperu.blogspot.com/2009/03/elrendimiento-academico_03.html

Stanovich, K. E., 1986. "Matthew effects in reading: Some consequences of individual differences in the acquisition of literacy". Reading Research Quarterly, Vol. 22, pp. 360-407.

Wigfield, A., 1994. "Expectancy-Value Theory of Achievement Motivation: A developmental perspective”. Educational Psychology Review, Vol. 6, No. 1, pp. 49-78.

Woods, D., Bruner, J. S. y Ross, G., 1976. "The role of tutoring in problem solving". Journal of Child Psychology and Psychiatry, Vol 17, No. 2, pp. 89-100. 
"LA REVISTA INNOVARE NO SE HACE RESPONSABLE EN NINGÚN CASO DE LOS CONTENIDOS, DATOS, CONCLUSIONES U OPINIONES VERTIDAS EN LOS ARTÍCULOS PUBLICADOS, SIENDO ESTA RESPOSABILIDAD EXCLUSIVA DEL (DE LOS) AUTOR (AUTORES)" 